\title{
Acceptance and commitment therapy for fatigue interference in advanced gastrointestinal cancer and caregiver burden: protocol of a pilot randomized controlled trial
}

\author{
Catherine E. Mosher ${ }^{1 *}$ D, Ekin Secinti ${ }^{1}$, Kurt Kroenke ${ }^{2}$, Paul R. Helft ${ }^{3}$, Anita A. Turk ${ }^{3}$, Patrick J. Loehrer Sr ${ }^{3}$, \\ Amikar Sehdev ${ }^{3}$, Ahmad A. Al-Hader ${ }^{3}$, Victoria L. Champion ${ }^{4}$ and Shelley A. Johns ${ }^{2}$
}

\begin{abstract}
Background: Fatigue interference with activities, mood, and cognition is one of the most prevalent and bothersome concerns of advanced gastrointestinal (Gl) cancer patients. As fatigue interferes with patient functioning, family caregivers often report feeling burdened by increasing responsibilities. Evidence-based interventions jointly addressing cancer patient fatigue interference and caregiver burden are lacking. In pilot studies, acceptance and commitment therapy (ACT) has shown promise for addressing symptom-related suffering in cancer patients. The current pilot trial seeks to test a novel, dyadic ACT intervention for both advanced Gl cancer patients with moderate-to-severe fatigue interference and their family caregivers with significant caregiving burden or distress.
\end{abstract}

Methods: A minimum of 40 patient-caregiver dyads will be randomly assigned to either the ACT intervention or an education/support control condition. Dyads in both conditions attend six weekly 50-min telephone sessions. Outcomes are assessed at baseline as well as 2 weeks and 3 months post-intervention. We will evaluate the feasibility, acceptability, and preliminary efficacy of ACT for improving patient fatigue interference and caregiver burden. Secondary outcomes include patient sleep interference and patient and caregiver engagement in daily activities, psychological flexibility, and quality of life. We will also explore the effects of ACT on patient and caregiver physical and mental health service use.

Discussion: Findings will inform a large-scale trial of intervention efficacy. Results will also lay the groundwork for further novel applications of ACT to symptom interference with functioning and caregiver burden in advanced cancer.

Trial Registration: ClinicalTrials.gov, NCT04010227. Registered 8 July 2019.

Keywords: Acceptance and commitment therapy, Fatigue, Symptom management, Metastatic gastrointestinal cancer, Family caregiver burden, Randomized controlled trial

\footnotetext{
* Correspondence: cemosher@iupui.edu

'Department of Psychology, Indiana University-Purdue University Indianapolis, 402 North Blackford Street, LD 124, Indianapolis, IN 46202, USA

Full list of author information is available at the end of the article
}

(c) The Author(s). 2021 Open Access This article is licensed under a Creative Commons Attribution 4.0 International License, which permits use, sharing, adaptation, distribution and reproduction in any medium or format, as long as you give appropriate credit to the original author(s) and the source, provide a link to the Creative Commons licence, and indicate if changes were made. The images or other third party material in this article are included in the article's Creative Commons licence, unless indicated otherwise in a credit line to the material. If material is not included in the article's Creative Commons licence and your intended use is not permitted by statutory regulation or exceeds the permitted use, you will need to obtain permission directly from the copyright holder. To view a copy of this licence, visit http://creativecommons.org/licenses/by/4.0/. The Creative Commons Public Domain Dedication waiver (http://creativecommons.org/publicdomain/zero/1.0/) applies to the data made available in this article, unless otherwise stated in a credit line to the data. 


\section{Background}

Gastrointestinal (GI) cancers (e.g., colorectal, esophageal, liver, pancreatic, stomach cancer) are among the most common cancers in the USA, and the majority are discovered at advanced stages [1]. Fatigue is a highly prevalent symptom, with up to $68 \%$ of advanced colorectal cancer patients reporting moderate-to-severe fatigue [2, 3]. Among GI cancer patients, fatigue often co-occurs with a number of symptoms and substantially impacts daily activities and quality of life (QoL) [3-7]. Among advanced cancer patients, fatigue and related symptoms have been linked to prolonged hospitalizations and readmissions [8].

As fatigue and related symptoms affect patient functioning, family caregivers cope with demanding role changes that may impact their mental and physical health [9-12]. Among caregivers of colorectal cancer patients, greater caregiving burden (i.e., impact of caregiving on various aspects of their lives) has been associated with impaired QoL [13]. This burden includes extensive time spent performing caregiving duties to the neglect of other vital activities, such as self-care [13-16].

Evidence-based interventions reducing both patient fatigue and caregiver burden in GI and other advanced cancers are lacking. Cochrane meta-analyses of pharmacologic and behavioral interventions for fatigue in advanced cancer patients characterized the evidence as inconclusive $[17,18]$. Additionally, behavioral interventions for cancer caregivers have produced small to medium effects on caregiving burden and QoL [19-21]. Most of these interventions were delivered to patientcaregiver dyads coping with early-stage cancer and did not have a symptom or distress criterion for study entry [19-21]. Thus, there is a significant need to develop interventions for symptomatic, advanced GI cancer patients and caregivers.

One behavioral intervention that shows potential for reducing symptom-related suffering in cancer and improving QoL is acceptance and commitment therapy (ACT) [22-25]. Rather than focusing on symptom reduction, the goal of ACT is to increase psychological flexibility so that unwanted symptoms, feelings, and thoughts interfere less with meaningful activities [26, 27]. Psychological flexibility is defined as fully experiencing the present moment while persisting in actions aligned with personal values [27]. In a pilot randomized controlled trial (RCT) in metastatic breast cancer, telephone-based ACT showed strong evidence of feasibility and promise for reducing fatigue interference with functioning compared to education/support [28]. ACT has also been found to reduce distress in various adult populations [29-31], although it has rarely been applied to caregivers of adults [32, 33].
The primary aim of this NIH-funded pilot RCT is to evaluate the feasibility and acceptability of delivering telephone-based ACT to advanced GI cancer patients and their caregivers. Our second aim is to test the potential effects of ACT on patient fatigue interference and caregiver burden (primary outcomes) as well as patient sleep interference and patient and caregiver engagement in daily activities, psychological flexibility, and QoL (secondary outcomes). We hypothesize that ACT will lead to improved outcomes as compared to education/support. This control condition involves supportive listening and education on medical center and community resources, consistent with common interventions in clinical settings. Our third aim is to explore the potential effects of ACT on patient and caregiver physical and mental health service use (tertiary outcomes). The current paper presents the rationale, design, methods, and analytic plan for this RCT.

\section{Methods/design \\ Overview of study design}

This pilot RCT examines the feasibility and acceptability of delivering telephone-based ACT to advanced GI cancer patient-caregiver dyads. Participants are recruited from the Indiana University Melvin and Bren Simon Comprehensive Cancer Center (IUSCCC) and Eskenazi Health in Indianapolis, Indiana. Figure 1 provides the enrollment, intervention, and assessment schedule. A minimum of 40 dyads will be randomized in equal numbers to six weekly 50-min telephone sessions of ACT or six weekly 50-min telephone sessions of education/support. Outcomes are assessed at baseline, 2 weeks postintervention (primary endpoint), and 3 months postintervention. ACT participants also complete qualitative interviews on ACT's acceptability. This trial was registered in ClinicalTrials.gov (NCT04010227) and approved by the IU Institutional Review Board. Any study amendments will be approved by the Institutional Review Board prior to implementation and will be reported to the trial registry. This protocol paper is prepared according to Standard Protocol Items: Recommendations for Interventional Trials (SPIRIT) guidelines (see Additional file 1: SPIRIT checklist).

\section{Patient and caregiver eligibility criteria}

Patient eligibility criteria are as follows: (a) $\geq 21$ years old (required by NIH program announcement); (b) at least 3 weeks post-diagnosis of unresectable stage III or stage IV GI cancer (i.e., anal, colon, esophageal, gallbladder, liver, pancreatic, rectal, small intestine, or stomach cancer) as ascertained through medical records and consultation with the attending oncologist; (c) has an eligible, consenting family caregiver; (d) no significant cognitive impairment as ascertained by medical record 


\begin{tabular}{|c|c|c|c|c|c|c|c|c|c|c|c|}
\hline \multirow[b]{3}{*}{ TIMEPOINT (week) } & \multirow{3}{*}{$\begin{array}{c}\begin{array}{c}\text { Enrollment/ } \\
\text { Screening }\end{array} \\
1\end{array}$} & \multirow{3}{*}{$\frac{\text { Baseline }}{2}$} & \multirow{3}{*}{\begin{tabular}{|c|} 
Allocation \\
2 \\
\end{tabular}} & \multicolumn{6}{|c|}{ STUDY PERIOD } & \multirow{3}{*}{\begin{tabular}{|c|}
$\begin{array}{c}2 \text { Weeks } \\
\text { Post-Intervention }\end{array}$ \\
10
\end{tabular}} & \multirow{3}{*}{\begin{tabular}{|c|}
$\begin{array}{c}\text { 3 Months } \\
\text { Post-Intervention }\end{array}$ \\
21 \\
\end{tabular}} \\
\hline & & & & \multicolumn{6}{|c|}{$\begin{array}{c}\text { Post-allocation } \\
\text { (Intervention) }\end{array}$} & & \\
\hline & & & & 3 & 4 & 5 & 6 & 7 & 8 & & \\
\hline \multicolumn{12}{|l|}{ ENROLMENT } \\
\hline \multirow{2}{*}{$\begin{array}{l}\text { Eligibility screening } \\
\text { Informed consent }\end{array}$} & $\mathrm{X}$ & & & & & & & & & & \\
\hline & $\mathrm{X}$ & & & & & & & & & & \\
\hline \multirow[t]{2}{*}{ Baseline assessment } & & $\mathrm{X}$ & & & & & & & & & \\
\hline & & & $\mathrm{X}$ & & & & & & & & \\
\hline \multicolumn{12}{|l|}{ INTERVENTIONS } \\
\hline \multicolumn{12}{|l|}{$\begin{array}{l}\text { Acceptance and Commitment Therapy } \\
(n=20 \text { dyads })\end{array}$} \\
\hline \multicolumn{12}{|l|}{ Education/Support ( $n=20$ dyads) } \\
\hline \multicolumn{12}{|l|}{ ASSESSMENTS* } \\
\hline \multicolumn{12}{|l|}{ 1. Feasibility: } \\
\hline \multirow{2}{*}{$\begin{array}{l}\text { Intervention acceptability: Intervention } \\
\text { acceptability items }(P, C) \\
\text { 2. Primary patient outcome: }\end{array}$} & & & & & & & & & & $\mathrm{X}$ & \\
\hline & & & & & & & & & & & \\
\hline $\begin{array}{l}\text { Fatigue interference: Fatigue interference } \\
\text { subscale of FSI }(P)\end{array}$ & & $\mathrm{x}$ & & & & & & & & $\mathrm{X}$ & $\mathrm{x}$ \\
\hline \multicolumn{12}{|l|}{ 3. Primary caregiver outcome: } \\
\hline \multirow{2}{*}{$\begin{array}{l}\text { Caregiving burden: Short form of Zarit } \\
\text { Burden Interview (C) } \\
\text { 4. Secondary outcomes: }\end{array}$} & & $\mathrm{X}$ & & & & & & & & $\mathrm{X}$ & $\mathrm{X}$ \\
\hline & & & & & & & & & & & \\
\hline \multirow{3}{*}{$\begin{array}{l}\text { Sleep interference: PROMIS short-form } \\
\text { sleep-related impairment measure }(P) \\
\text { Engagement in daily activities: } P R O M I S \\
\text { short-form measure of ability to participate } \\
\text { in social roles and activities, Values } \\
\text { Progress subscale of Valuing Questionnaire } \\
(P, C)\end{array}$} & & $\mathrm{X}$ & & & & & & & & $\mathrm{X}$ & $\mathrm{X}$ \\
\hline & & $\mathrm{X}$ & & & & & & & & $\mathrm{X}$ & $\mathrm{X}$ \\
\hline & & & & & & & & & & & \\
\hline \multirow{3}{*}{$\begin{array}{l}\text { Psychological flexibility: Acceptance and } \\
\text { Action Questionnaire-II (P, C) } \\
\text { Quality of life: McGill Quality of Life } \\
\text { Questionnaire-Revised (P), PROMIS Global } \\
\text { Health measure (C) } \\
\text { 5. Tertiary outcomes: }\end{array}$} & & $\mathrm{X}$ & & & & & & & & $\mathrm{X}$ & $\mathrm{X}$ \\
\hline & & $\mathrm{X}$ & & & & & & & & $\mathrm{X}$ & $\mathrm{X}$ \\
\hline & \multicolumn{11}{|c|}{ 5. Tertiary outcomes: } \\
\hline \multirow{2}{*}{$\begin{array}{l}\text { Physical and mental healthcare use: } \\
\text { Healthcare use interview, medication } \\
\text { interview }(P, C) \\
\text { 6. Demographic and medical factors: }\end{array}$} & & $\mathrm{X}$ & & & & & & & & $\mathrm{X}$ & $\mathrm{X}$ \\
\hline & & & & & & & & & & & \\
\hline Sociodemographics $(P, C)$ & & $\mathrm{X}$ & & & & & & & & & \\
\hline $\begin{array}{l}\text { Medical comorbidity: Checklist of } 8 \\
\text { conditions }(P) \text { or } 9 \text { conditions }(C)\end{array}$ & & $\mathrm{X}$ & & & & & & & & & \\
\hline $\begin{array}{l}\text { Functional status: Patient-reported ECOG } \\
\text { score }(P)\end{array}$ & & $\mathrm{X}$ & & & & & & & & $\mathrm{X}$ & $\mathrm{X}$ \\
\hline $\begin{array}{l}\text { Cancer information (e.g., date of diagnosis, } \\
\text { cancer treatments): Chart review }\end{array}$ & & $\mathrm{X}$ & & & & & & & & $\mathrm{X}$ & $\mathrm{X}$ \\
\hline 7. Severity of symptoms: & & & & & & & & & & & \\
\hline $\begin{array}{l}\text { Fatigue severity and frequency: Fatigue } \\
\text { severity and frequency items from } F S I(P, C)\end{array}$ & & $\mathrm{X}$ & & & & & & & & $\mathrm{X}$ & $\mathrm{X}$ \\
\hline $\begin{array}{l}\text { Sleep disturbance: PROMIS short-form } \\
\text { measure of sleep disturbance }(P, C)\end{array}$ & & $\mathrm{X}$ & & & & & & & & $\mathrm{X}$ & $\mathrm{X}$ \\
\hline $\begin{array}{l}\text { Cognitive concerns: PROMIS short-form } \\
\text { measure of cognitive concerns }(P, C)\end{array}$ & & $\mathrm{X}$ & & & & & & & & $\mathrm{X}$ & $\mathrm{x}$ \\
\hline $\begin{array}{l}\text { Anxiety: PROMIS short-form measure of } \\
\text { anxiety }(P, C)\end{array}$ & & $\mathrm{X}$ & & & & & & & & $\mathrm{X}$ & $\mathrm{X}$ \\
\hline $\begin{array}{l}\text { Depressive symptoms: } P R O M I S \text { short-form } \\
\text { measure of depressive symptoms }(P, C)\end{array}$ & & $\mathrm{X}$ & & & & & & & & $\mathrm{X}$ & $\mathrm{X}$ \\
\hline $\begin{array}{l}\text { Pain severity and interference: PROMIS } \\
\text { short-form measures of pain severity and } \\
\text { interference }(P)\end{array}$ & & $\mathrm{X}$ & & & & & & & & $\mathrm{X}$ & $\mathrm{X}$ \\
\hline
\end{tabular}

Fig. 1 Schedule of enrollment, interventions, and assessments (Standard Protocol Items: Recommendations for Interventional Trials) 
review and a mental status questionnaire [34]; (e) English fluency; ( $\mathrm{f}$ ) working phone service; ( $\mathrm{g}$ ) in bed or chair less than half the day (patient-reported Eastern Cooperative Oncology Group [ECOG] score $\leq 2$ ) [35]; (h) not enrolled in hospice; and (i) moderate-to-severe fatigue interference (mean score $\geq 2.5$ on the Fatigue Interference subscale of the Fatigue Symptom Inventory [FSI]) $[36,37]$. The clinical validity of this cut point is supported by our pilot research with advanced cancer patients. Caregiver eligibility criteria are as follows: (a) $\geq$ 18 years of age, (b) family caregiver who either lives with the patient or has visited them at least twice a week for the past month, (c) English fluency, (d) working phone service, and (e) significant caregiving burden (score $\geq 6$ on the 6-item Zarit Burden Interview) [38] or distress on the 4-item Patient-Reported Outcomes Measurement Information System (PROMIS) anxiety or depression measures ( $T$-score $\geq 60$ [at least one standard deviation above the population mean] for anxiety or depression) [39]. The clinical cutoff for the Zarit Burden Interview has been established in advanced cancer caregivers [38]. We administer anxiety and depression measures at screening in order to enroll caregivers with high distress who may not endorse caregiving burden due to cultural factors.

\section{Recruitment}

Initial patient eligibility is determined via electronic health record review and consultation with the attending oncologist. Prior to the COVID-19 pandemic, trained research assistants (RAs) approached patients and caregivers during oncology clinic visits or via phone. After COVID-19 restrictions were implemented, potential participants have only been approached through recruitment mailings and phone calls. The mailings include a consent form and letter of invitation with a phone number for opting out of further contact. An RA calls all patients who do not opt out approximately 1 to 2 weeks after the letter is mailed. The RA reviews the consent form in detail and answers questions. Interested patients identify a family caregiver and are screened for eligibility. Eligible and interested patients provide consent for study participation over the phone under a waiver of documentation of written informed consent. Caregivers of consenting patients receive a consent form via email or postal mail followed by telephone calls for eligibility screening and consent. With the patient's permission, up to three of their family caregivers are consecutively screened for eligibility. If none of the caregivers are eligible and consent to participate, then the patient is ineligible for study participation.

\section{Randomization and blinding}

Following baseline assessments, dyads are randomly assigned in equal numbers to ACT or education/support using stratified block randomization to balance the groups by performance status (patient-reported ECOG scores 0 or 1 vs. 2) [35]. Performance status informs treatment decisions in advanced GI cancer, such as whether to provide chemotherapy [40]. A study statistician used the R package "blockrand" $[41,42]$ to generate randomly varying block sizes of $2,4,6$, and 8 . Following randomization, study condition assignments are identifiable to participants, interventionists, and individuals mailing intervention materials. Other investigators, outcome assessors, and data analysts are blinded throughout the trial. Strategies to reduce the potential for unblinding include storing study condition assignments in separate data sheets that are only available to unblinded individuals. Additionally, the RA asks participants to refrain from discussing the intervention during follow-up assessments.

\section{Intervention \\ Acceptance and commitment therapy}

We developed the ACT manual, which was informed by literature on the experiences of advanced GI cancer patients and caregivers [2, 3, 14, 43, 44], the ACT model $[22,27]$, previous ACT trials with cancer patients and other medical populations $[25,26,28,29,31,45,46]$, and our clinical experience. A summary of intervention components is found in Table 1. Grounded in the ACT model [22, 27], the intervention is designed to reduce patient fatigue interference and caregiver burden by increasing psychological flexibility (see Fig. 2: conceptual model). Psychological flexibility is expected to increase through practicing mindfulness (e.g., meditations that encourage non-judgmental awareness of thoughts, feelings, and bodily sensations in the present moment), learning adaptive coping skills (e.g., acceptance, cognitive defusion, perspective-taking), identifying personal values (e.g., being a loving partner), and setting specific goals aligned with these values. Value-based action goals are in the SMART format (specific, measurable, achievable, relevant, and time-bound).

Patients and caregivers complete sessions 1 and 4-6 together via speakerphone. Sessions 2 and 3 are delivered to patients and caregivers separately. Holding both dyadic and individual sessions allows the therapist to meet the shared and unique needs of patients and caregivers. We adapted ACT to the dyad by incorporating joint mindfulness practices and leveraging the relationship during discussions. For example, participants discuss moments of emotional connection with each other as instances of mindfulness and provide encouragement to the other person during goal setting. Individual 
Table 1 Summary of core components of each intervention condition

\section{Acceptance and commitment therapy}

- Discuss current attempts to control fatigue (if patient) or difficult thoughts and feelings (if caregiver) and their impact on quality of life -Practice mindfulness with the therapist during sessions and at home (e.g., awareness of the breath, body scan, leaves on a stream)

-Practice cognitive defusion-noticing symptoms, thoughts, and feelings without being overwhelmed by them (e.g., passengers on the bus metaphor)

- Observe and detach from difficult internal experiences (e.g., fatigue, thoughts, feelings) to cultivate a transcendent sense of self from which to notice and accept changing experiences

-Identify important values (e.g., being a loving and engaged parent, serving one's community)

-Goal setting and practice of value-based actions

\section{Education/support}

- Orientation to the medical center and treatment team; provide overview of quality-of-life concerns and discuss physical quality of life and symptoms

-Discuss cancer-related social challenges (e.g., talking with children about cancer, employment concerns); tips on managing the household when ill; describe resources for addressing social challenges

-Discuss common emotional reactions to cancer, including anxiety, depression, and stress, and cancer-related cognitive changes and describe available mental health services

- Review common financial challenges related to cancer and its treatment and describe resources for addressing these challenges

-Discuss methods of evaluating health information on the Internet and other modalities

- Review previous session topics and refer to websites with cancer information

sessions with patients and caregivers include exploration of personal values. In addition, the workability of attempts to avoid fatigue (for patients) or unwanted thoughts and feelings about caregiving (for caregivers) is discussed. Thus, the focus of individual sessions is shifting from avoidant responses to the pursuit of valuebased action despite fatigue or perceived caregiving burden.

During each session, patients complete three items from the FSI (i.e., average fatigue and fatigue interference with general activity and enjoyment of life) [36, 37], and both patients and caregivers complete two PROMIS anxiety items and two PROMIS depression items [39]. These reports allow the therapist to monitor participant fatigue and distress. Each participant's home practice of mindfulness and other skills is also recorded, and practice for the week ahead is discussed. Each person receives handouts summarizing session topics and audio recordings that our team developed to guide mindfulness practices.

\section{Education/support}

Dyads in the education/support arm are directed to resources for practical and health information and contact information for psychosocial services. This condition controls for time and attention given to participants as well as therapist empathy, active listening, and education about resources. A similar comparator was used in our ACT pilot trial with metastatic breast cancer patients [28], and similar comparison groups have been used in studies with primarily advanced cancer patient-caregiver dyads [33, 47, 48]. The duration of sessions and number and order of dyadic vs. individual sessions are identical to those of the ACT condition. In addition, the same fatigue, depression, and anxiety assessments as those in the ACT condition are completed during each session $[36,37,39]$. A summary of the topics for each education/support session is found in Table 1. Education/support participants receive handouts on session topics and are asked to review them as homework. ACT concepts are not discussed.

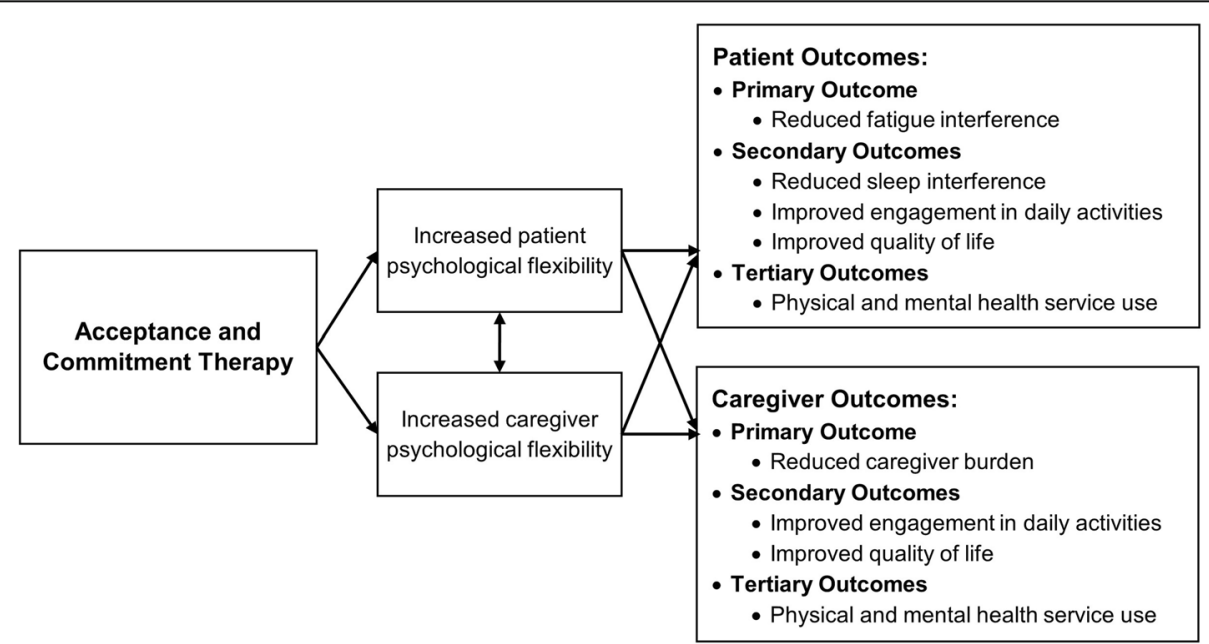

Fig. 2 Conceptual model. Although psychological flexibility is a secondary outcome rather than a mediator in this pilot trial, our analyses will set the stage for formal mediation analyses in a future large-scale trial 


\section{Training of study staff and treatment fidelity}

Training of interventionists and RAs involved didactics, live demonstrations, and role-plays. All assessments, interviews, and intervention sessions are digitally recorded. The principal investigator and trained doctoral students in clinical psychology are randomly reviewing $20 \%$ of assessments and qualitative interviews for adherence to the protocol.

Interventionists are doctoral level psychologists or master's level mental health clinicians with extensive training and experience delivering ACT or supportive counseling. Each intervention is delivered by different therapists to prevent cross-contamination between study conditions. Interventionists received initial education in advanced GI cancer diagnosis and treatment, distress, and either ACT or supportive counseling techniques with training protocols developed in our team's prior studies. Initial training also involved role-plays of sessions detailed in manuals. Licensed psychologists and doctoral students in clinical psychology review a random selection of recordings for adherence to the manual using checklists from our pilot research. All fidelity monitors have expertise in ACT or supportive counseling techniques and received training in the intervention protocol. Psychologists review sessions from both study conditions, whereas the doctoral students only review education/support sessions. During regular supervisory meetings with study therapists, psychologists provide treatment adherence scores (number of required topics and exercises covered in each session/total number of fidelity criteria) and discuss treatment fidelity issues.

\section{Retention}

Several strategies are employed to enhance participant retention. All study staff, including RAs and therapists, have been instructed to clearly state expectations to participants and offer the option of completing follow-up assessments if they decide to discontinue the intervention. The use of the telephone for the entire study, including assessments, intervention sessions, and qualitative interviews, accommodates rural residents and those with low incomes. Participants are reminded of appointments via text, phone, or email. Finally, each person is mailed $\$ 40$ in gift cards to a major retailor after completion of each assessment or interview.

\section{Adverse events and auditing}

If any adverse events occur, such as increased risk of suicidality, a licensed mental health provider on the study team will refer the participant to mental health services. Adverse events will be reported to the Clinical Trial Management Center at IUSCCC comprised of experienced clinical researchers and clinicians and to the IU Institutional Review Board. All trials conducted at the
IUSCCC are subject to quarterly audit by the Clinical Trial Management Center, which serves as an independent data monitoring committee.

\section{Study measures and data collection schedule}

Figure 1 shows the study measures and the data collection schedule. Assessments are conducted by RAs blind to study condition. Patients complete a 30-min baseline assessment and two 25-min follow-up assessments. Caregivers complete a 25-min baseline assessment and two 20-min follow-up assessments. All measures have evidence of reliability and validity. Within approximately 2 weeks of the first follow-up, ACT participants are invited to complete a separate 30-min qualitative interview about ACT's acceptability. Qualitative interviews are conducted with patients and caregivers separately.

\section{Feasibility and acceptability data}

Feasibility is assessed by accrual rates, attrition, and adherence (i.e., session attendance in both study conditions, home practice completion for ACT participants). Reasons for non-adherence to the intervention and study withdrawal are recorded. At the first follow-up, acceptability of both study conditions is assessed with helpfulness ratings on a scale from 1 (did not help at all) to 5 (extremely helpful) for number and length of sessions, topics, therapist, and telephone format [49]. Participants also rate the extent to which the telephone sessions met their expectations on a scale from 1 (not at all) to 5 (extremely). The RA is blind to study condition, as the questions apply to either condition. For ACT participants, acceptability is also assessed via qualitative interviews on the perceived helpfulness and impact of ACT components (see Additional file 2 for interview protocol).

\section{Primary patient-centered outcomes}

The primary outcome measure for patients is the 7-item Fatigue Interference subscale of the FSI [36, 37]. Items assess the extent to which fatigue in the past week interfered with activities, such as bathing, dressing, and housework, ability to concentrate, enjoyment of life, and mood on 11-point scales ( $0=$ no interference; $10=$ extreme interference). The primary outcome measure for caregivers is the 12-item short form of the Zarit Burden Interview [38, 50], which evaluates personal strain and role strain due to caregiving on 5-point scales $(0=$ never; $4=$ nearly always).

\section{Secondary outcomes}

(1) Patient sleep interference is assessed using the 8-item PROMIS sleep-related impairment measure, which evaluates perceived interference of sleep problems with activities, mood, and cognition [51, 52]. (2) Patient and 
caregiver engagement in daily activities is assessed with the 6-item PROMIS measure of ability to participate in social roles and activities [53]. The items, which are reverse-coded, measure difficulty engaging in social and recreational activities as well as usual work (including housework). Patients and caregivers also report their progress in living consistently with personal values by completing the 5-item Value Progress subscale of the Valuing Questionnaire [54]. (3) Patient and caregiver psychological flexibility is evaluated with the 7-item Acceptance and Action Questionnaire-II (AAQ-II) [55]. (4) Patient $Q o L$ is assessed with the 15-item McGill Quality of Life Questionnaire-Revised, which was designed for patients with life-threatening illnesses and evaluates physical, existential, and social well-being [56]. Caregiver QoL is assessed with the 10-item PROMIS measure of global health, including physical, mental, and social wellbeing [57].

\section{Tertiary outcomes}

Patients and caregivers report their physical and mental healthcare use in five domains (e.g., ER visits, outpatient visits) in the past 3 months at baseline and over the study period $[58,59]$. These reports of healthcare use were sensitive to change in a cancer trial [60]. At all time points, participants also report whether professionals referred them to support services and whether referred services were received $[61,62]$. Additionally, at all time points, participants report current medications using established methods from prior trials [63, 64]. We will compute the total number of medications at each time point, as this variable had strong predictive validity for both healthcare use and costs in older adults over a 1year period [65].

\section{Descriptive variables}

Patient and caregiver demographics and medical factors are assessed to characterize the sample. Caregivers also report their relationship to the patient. Patients and caregivers complete a checklist of eight or nine chronic health conditions, respectively [59]. Patient functional status is also assessed with the 1-item Patient Generated Subjective Global Assessment (PG-SGA), a patientreported version of the ECOG score [35]. Patient cancer information, such as time since diagnosis and treatments, is collected via chart review. Finally, the severity of patient and caregiver symptoms is assessed. For both patients and caregivers, fatigue severity and frequency are measured with six items from the FSI [36, 37], and sleep disturbance, cognitive concerns, anxiety, and depressive symptoms are each assessed with a 6-item PROMIS measure [39, 51, 52, 66-68]. Patient pain is evaluated with 3-item and 4-item PROMIS measures of severity and interference, respectively $[66,69]$.

\section{Statistical analyses}

\section{Data management}

Paper copies of assessments and other study materials are stored in locked filing cabinets in locked offices. Electronic data are kept in password-protected files on the secure, HIPAA-compliant IU server. Only IRBapproved members of the research team have access to the data. Participant identifiers are stored separately from participant data in a locked office or secure electronic files. Data are coded by participant identification number. Data are entered into the secure study database in REDCap and checked by two RAs with the supervision of the principal investigator. The principal investigator and data analysts will have access to the cleaned data set.

\section{Preliminary analyses}

All data will be assessed for missingness and multiple imputation with 50 imputed samples will be used [70]. We will also compare participants and those who decline participation or withdraw on demographic and medical factors using t-tests and Chi-square analyses.

We will assess for differences in baseline factors (e.g., demographic, medical and healthcare use variables) between study conditions [71]. Any differences will be considered when interpreting findings, and factors that vary between the groups will be included as covariates in sensitivity analyses.

\section{Analysis for aim 1}

Our analysis plan for aim 1 focuses on the feasibility and acceptability of the ACT intervention. Feasibility will be assessed by accrual rates, attrition, and adherence. We will judge this trial as feasible if (1) at least $60 \%$ of screened eligible dyads enroll in the study [72] and (2) at least $70 \%$ of randomized dyads complete 5-6 intervention sessions and the 2-week follow-up. These benchmarks for feasibility are based on our own pilot work and other published trials with advanced cancer patients and caregivers $[19,20,72]$. Acceptability of ACT and education/support sessions will be descriptively assessed with ratings of the helpfulness of the number and length of the sessions, topics, therapist, and telephone format on a scale from 1 (did not help at all) to 5 (extremely helpful) [49]. We will judge this trial as acceptable if at least $70 \%$ of patients and caregivers rate ACT as moderately to extremely helpful (i.e., an average score $\geq 4$ on 1 to 5 Likert scale items). We will also descriptively examine whether the ACT and education/support sessions met patients' and caregivers' expectations on a scale from 1 (not at all) to 5 (extremely). Additionally, we will conduct descriptive within-dyad comparisons of all acceptability ratings. 
For ACT participants, acceptability will also be assessed with semi-structured qualitative interviews on the perceived helpfulness of intervention components and their impact on functioning and well-being. The interviews will be audio-recorded, transcribed verbatim, and analyzed using an immersion/crystallization approach [73]. A clinical psychologist and health communication specialist, along with the RA, will develop a coding system. The analysis will consist of two phases: open and focused coding [73, 74]. Open coding facilitates the development of a code list for further analysis. In this phase, the analysts will independently label each line of data to reflect meanings or themes emerging from the text. This is done iteratively, combining, adding, or eliminating themes, until analysts agree on a set of emergent thematic categories (codes). In focused coding, codes derived in open coding are independently applied to all transcripts. To ensure that analysts remain consistent with coding, every fourth transcript will be coded by all three analysts, who will meet to discuss and reach consensus on coding. Atlas-ti software will facilitate coding, and qualitative results will inform intervention refinement for the subsequent large-scale RCT.

\section{Analysis for aim 2}

Given that this is a pilot study, our data analytic approach will be to derive effect size estimates rather than test for statistical significance. Effect sizes will be cautiously interpreted, as they are likely to be biased and imprecise [75]. A linear mixed model repeated measures approach (SAS Proc-Mixed) will be used to examine ACT's effects on primary and secondary outcomes. For outcome measures that only patients or caregivers complete (e.g., patient fatigue interference or caregiver burden), models will include main effects of time (as categorical) and study group and the time-by-study group interaction. Treatment effects will be evidenced by the interaction between time and study group (i.e., group mean differences after the intervention but no such differences at baseline). Models will include random intercepts to account for nonindependence of individuals' scores across time. For outcomes reported by patients and caregivers (e.g., psychological flexibility), multilevel modeling for dyadic data will be used [76, 77]. Models will include the main effects of time, study group, and social role (patient vs. caregiver) as well as all two- and three-way interactions between these variables. The time $\times$ study group $\times$ role interaction will estimate the degree to which treatment effects are different for patients and caregivers. Dyadic models will include random intercepts for patients and caregivers, as well as the covariance between the intercepts to account for nonindependence across time and across partners.
We plan to recruit at least 40 advanced GI cancer patients and 40 caregivers, consistent with the recommendations of the Stage Model of Behavior Therapies Research for examining feasibility and effect sizes in a pilot trial [78]. Although our analyses focus on effect sizes rather than statistical significance, we calculated power for 34 patients and 34 caregivers at 2 weeks postintervention (assuming a 15\% attrition rate) [49]. For each of the primary outcomes (patient fatigue interference and caregiver burden), we will have $80 \%$ power ( $p$ $=.05$, two-tailed) to detect a large intervention effect $(d$ $=.99$ ) in a linear mixed model [79]. We expect medium to large effects of ACT on primary and secondary outcomes based on the literature. For example, ACT had large effects on distress and QoL outcomes in late-stage ovarian cancer patients $(d \mathrm{~s}=.89-1.69)$ compared to cognitive-behavioral therapy [25] and a medium effect ( $d$ $=-.59$ ) on fatigue interference compared to education/ support among metastatic breast cancer patients with moderate-to-severe baseline fatigue interference in our pilot trial [28]. We do not have preliminary data to estimate an effect of ACT on cancer caregiver burden.

\section{Analysis for aim 3}

We will use logistic and Poisson regression models to explore the effects of ACT on patient and caregiver physical and mental health service use (e.g., number of patient ER visits, patient and caregiver use of counseling and/or psychiatric medication). Logistic and Poisson regression analyses are appropriate for binary and count outcome data, respectively. Analyses will examine study condition as a predictor of health service use over the entire study period, controlling for baseline service use. Support service referrals will be a covariate in analyses of mental health service use.

\section{Discussion}

The present pilot trial addresses the top-rated concern of advanced GI cancer patients-fatigue's interference with activities, mood, and cognition [80] — and family caregiver burden. Evidence-based interventions to address these concerns are lacking. The ACT model [27] and pilot studies of ACT with cancer patients [24-26, $28,45,46]$ provide a strong rationale for the current trial. This project will be a catalyst for addressing a critical gap in the evidence-based care of this large, understudied population.

ACT is hypothesized to reduce fatigue interference in cancer patients, as it emphasizes mindful acceptance of the present moment-including internal experiences such as fatigue-and engagement in activities aligned with personal values. Our pilot trial with metastatic breast cancer patients was the first to examine ACT's effect on fatigue interference [28]. The intervention 
showed strong evidence of feasibility and promise regarding its impact on this outcome [28]. This study expands this line of research to both advanced GI cancer patients with significant fatigue interference and their caregivers. Of note, the use of a fatigue interference eligibility criterion for patients and a caregiver burden/distress criterion for caregivers allows us to focus on dyads with greater need for intervention, an approach rarely used in the broader psycho-oncology literature $[19,81]$.

The current ACT intervention is one of the first to use a dyadic approach [33]. Although preliminary evidence suggests that ACT improves QoL outcomes in patients with cancer and other chronic conditions $[25,26,28,29$, $31,45]$, these trials have almost exclusively focused on patients. Because caregivers often experience significant burden [13, 14], evidence-based interventions should be developed to improve their QoL and capacity to balance important roles and activities. A dyadic intervention may improve outcomes for both patients and caregivers as they reinforce each other's practice of ACT skills. Additionally, the intervention will capitalize on the relational bond between family members. For example, when setting personal goals, each family member will describe the strengths and resources that the other person possesses to facilitate goal achievement. Thus, our dyadic ACT intervention aims to improve interdependent QoL and behavioral outcomes by leveraging the familial relationship.

Our ability to demonstrate the feasibility and promise of ACT will lead to a large-scale trial and ultimately fulfill an unmet need in the comprehensive care of advanced GI cancer patients and caregivers. Given widely available training in $\mathrm{ACT}$, the intervention can be readily disseminated to clinicians. Results will also provide a foundation for a program of research focused on the novel application of ACT to symptom interference with functioning and caregiver burden in advanced cancer.

Results of this study will be published in a peerreviewed journal and submitted to ClinicalTrials.gov within 12 months of the primary completion date. The trial protocol and data set will be available to qualified investigators within 6 months of the publication of the primary outcome paper. The investigators will be required to sign a data use agreement and obtain IRB approval before receiving the data set.

\footnotetext{
Abbreviations

AAQ-II: Acceptance and Action Questionnaire-II; ACT: Acceptance and commitment therapy; ECOG: Eastern Cooperative Oncology Group; FSI: Fatique Symptom Inventory; GI: Gastrointestinal; PG-SGA: Patient Generated Subjective Global Assessment; PROMIS: Patient-Reported Outcomes Measurement Information System; QoL: Quality of life; RA: Research assistant; RCT: Randomized controlled trial; SMART: Specific, measurable, achievable, relevant, and time-bound
}

\section{Supplementary Information}

The online version contains supplementary material available at https://doi. org/10.1186/s40814-021-00837-9

Additional file 1:. SPIRIT checklist

Additional file 2:. Qualitative interview protocols for patients and caregivers

\section{Acknowledgements}

We thank the medical staff at study sites, Kelly Chinh, M.S.; Ellen Krueger, M.S.; Ashley B. Lewson, B.S.; Isabella Stuart, B.S.; Miriam Wright; Michelle Hoy, LCSW, OSW-C, CHWC; and Thomas Parry, M.S., M.Ed., BCBA, LBA, for their contributions to this project.

\section{Authors' contributions}

CEM, the principal investigator, developed the intervention and trial design, obtained funding, developed the study protocol and current manuscript, and oversees all study procedures. ES assisted in writing the manuscript. KK assisted in the trial design and application for funding. PRH, AAT, PJL, AS, and AAA helped in the trial design and assist in patient recruitment. VLC assisted in the trial design and application for funding. SAJ assisted with developing the intervention, trial design, and application for funding and oversees study therapists. All authors read and approved the final manuscript.

\section{Funding}

This work was supported by the National Cancer Institute [R21CA235788] The National Cancer Institute was not involved in the study design, the writing of the report, or the decision to submit the article for publication and does not have ultimate authority over these activities.

Availability of data and materials

Not applicable

\section{Declarations}

Ethics approval and consent to participate

Ethics approval of this protocol was granted by the Indiana University Institutional Review Board (protocol no. 1904388865). The trial is being conducted in compliance with the Declaration of Helsinki. Informed consent will be obtained from all participants.

Consent for publication

Not applicable

Competing interests

The authors declare that they have no competing interests.

\section{Author details}

${ }^{1}$ Department of Psychology, Indiana University-Purdue University Indianapolis, 402 North Blackford Street, LD 124, Indianapolis, IN 46202, USA. ${ }^{2}$ Indiana University School of Medicine, Center for Health Services Research, Regenstrief Institute, 1101 W. 10th Street, Indianapolis, IN 46202, USA.

${ }^{3}$ Indiana University School of Medicine, Indiana University Melvin and Bren Simon Comprehensive Cancer Center, Indiana Cancer Pavilion, 535 Barnhill Drive, Suite 473, Indianapolis, IN 46202, USA. ${ }^{4}$ Indiana University School of Nursing, 1111 Middle Drive, NU 340G, Indianapolis, IN 46202, USA.

Received: 10 October 2020 Accepted: 10 April 2021

Published online: 20 April 2021

\section{References}

1. American Cancer Society. Cancer facts and figures 2020. Atlanta: American Cancer Society; 2020.

2. Walling AM, Weeks JC, Kahn KL, Tisnado D, Keating NL, Dy SM, et al. Symptom prevalence in lung and colorectal cancer patients. J Pain Symptom Manage. 2015:49(2):192-202. https://doi.org/10.1016/j.jpainsymma n.2014.06.003 
3. Vardy J, Dhillon HM, Pond GR, Rourke SB, Xu W, Dodd A, et al. Cognitive function and fatigue after diagnosis of colorectal cancer. Ann Oncol. 2014; 25(12):2404-12. https://doi.org/10.1093/annonc/mdu448.

4. Breitbart W, Rosenfeld B, Tobias K, Pessin H, Ku GY, Yuan J, et al. Depression, cytokines, and pancreatic cancer. Psychooncology. 2014;23(3):339-45. https://doi.org/10.1002/pon.3422.

5. Husson O, Mols F, van de Poll-Franse LV, Thong MS. The course of fatigue and its correlates in colorectal cancer survivors: a prospective cohort study of the PROFILES registry. Support Care Cancer. 2015;23(11):3361-71. https:// doi.org/10.1007/s00520-015-2802-x.

6. Mota DD, Pimenta CA, Caponero R. Fatigue in colorectal cancer patients: prevalence and associated factors. Rev Lat Am Enfermagem. 2012;20(3):495503. https://doi.org/10.1590/S0104-11692012000300010.

7. Farkkila N, Sintonen $\mathrm{H}$, Saarto $\mathrm{T}$, Jarvinen $\mathrm{H}$, Hanninen J, Taari $\mathrm{K}$, et al. Health-related quality of life in colorectal cancer. Colorectal Dis. 2013;15(5): e215-22. https://doi.org/10.1111/codi.12143

8. Nipp RD, El-Jawahri A, Moran SM, D'Arpino SM, Johnson PC, Lage DE, et al. The relationship between physical and psychological symptoms and health care utilization in hospitalized patients with advanced cancer. Cancer. 2017; 123(23):4720-7. https://doi.org/10.1002/cncr.30912.

9. Engebretson A, Matrisian L, Thompson C. Pancreatic cancer: Patient and caregiver perceptions on diagnosis, psychological impact, and importance of support. Pancreatology. 2015;15(6):701-7. https://doi.org/10.1016/j.pan.2 015.05.471.

10. Janda M, Neale RE, Klein K, O'Connell DL, Gooden H, Goldstein D, et al. Anxiety, depression and quality of life in people with pancreatic cancer and their carers. Pancreatology. 2017;17(2):321-7. https://doi.org/10.1016/j.pan.2 017.01.008.

11. Shaffer KM, Jacobs JM, Nipp RD, Carr A, Jackson VA, Park ER, et al. Mental and physical health correlates among family caregivers of patients with newly-diagnosed incurable cancer: a hierarchical linear regression analysis. Support Care Cancer. 2017;25(3):965-71. https://doi.org/10.1007/s00520-01 6-3488-4.

12. Wadhwa D, Burman D, Swami N, Rodin G, Lo C, Zimmermann C. Quality of life and mental health in caregivers of outpatients with advanced cancer. Psychooncology. 2013;22(2):403-10. https://doi.org/10.1002/pon.2104.

13. Hanly P, Maguire R, Hyland P, Sharp L. Examining the role of subjective and objective burden in carer health-related quality of life: the case of colorectal cancer. Support Care Cancer. 2015;23(7):1941-9. https://doi.org/10.1007/ s00520-014-2551-2

14. van Ryn M, Sanders S, Kahn K, van Houtven C, Griffin JM, Martin M, et al. Objective burden, resources, and other stressors among informal cancer caregivers: a hidden quality issue? Psychooncology. 2011;20(1):44-52. https://doi.org/10.1002/pon.1703.

15. Yabroff KR, Kim Y. Time costs associated with informal caregiving for cancer survivors. Cancer. 2009;115(18 Suppl):4362-73. https://doi.org/10.1002/ cncr.24588.

16. Maguire R, Hanly $P$, Hyland $P$, Sharp L. Understanding burden in caregivers of colorectal cancer survivors: what role do patient and caregiver factors play? Eur J Cancer Care (Engl). 2018;27(1):e12527. https://doi.org/10.1111/ ecc.12527.

17. Mucke M, Cuhls H, Peuckmann-Post V, Minton O, Stone P, Radbruch L. Pharmacological treatments for fatigue associated with palliative care. Cochrane Database Syst Rev. 2015;5:CD006788.

18. Poort H, Peters M, Bleijenberg G, Gielissen MF, Goedendorp MM, Jacobsen $P$, et al. Psychosocial interventions for fatigue during cancer treatment with palliative intent. Cochrane Database Syst Rev. 2017;7:Cd012030.

19. Northouse LL, Katapodi MC, Song L, Zhang L, Mood DW. Interventions with family caregivers of cancer patients: meta-analysis of randomized trials. CA Cancer J Clin. 2010;60(5):317-39. https://doi.org/10.3322/caac.20081.

20. Badr $\mathrm{H}$, Krebs P. A systematic review and meta-analysis of psychosocial interventions for couples coping with cancer. Psychooncology. 2013;22(8): 1688-704. https://doi.org/10.1002/pon.3200

21. O'Toole MS, Zachariae R, Renna ME, Mennin DS, Applebaum A. Cognitive behavioral therapies for informal caregivers of patients with cancer and cancer survivors: a systematic review and meta-analysis. Psychooncology. 2017;26(4):428-37. https://doi.org/10.1002/pon.4144.

22. Hayes SC, Strosahl KD, Wilson KG. Acceptance and commitment therapy, second edition: the process and practice of mindful change. New York: Guilford Press; 2012
23. Hulbert-Williams NJ, Storey L, Wilson KG. Psychological interventions for patients with cancer: psychological flexibility and the potential utility of acceptance and commitment therapy. Eur J Cancer Care (Engl). 2015;24(1): 15-27. https://doi.org/10.1111/ecc.12223.

24. Arch JJ, Fishbein JN, Ferris MC, Mitchell JL, Levin ME, Slivjak ET, et al. Acceptability, feasibility, and efficacy potential of a multimodal acceptance and commitment therapy intervention to address psychosocial and advance care planning needs among anxious and depressed adults with metastatic cancer. J Palliat Med. 2020;23(10):1380-5. https://doi.org/10.1089/ jpm.2019.0398.

25. Rost AD, Wilson K, Buchanan E, Hildebrandt MJ, Mutch D. Improving psychological adjustment among late-stage ovarian cancer patients: examining the role of avoidance in treatment. Cogn Behav Pract. 2012;19(4): 508-17. https://doi.org/10.1016/j.cbpra.2012.01.003.

26. Feros DL, Lane L, Ciarrochi J, Blackledge JT. Acceptance and commitment therapy $(A C T)$ for improving the lives of cancer patients: a preliminary study. Psychooncology. 2013;22(2):459-64. https://doi.org/10.1002/pon.2083.

27. Hayes SC, Levin ME, Plumb-Vilardaga J, Villatte JL, Pistorello J. Acceptance and commitment therapy and contextual behavioral science: examining the progress of a distinctive model of behavioral and cognitive therapy. Behav Ther. 2013;44(2):180-98. https://doi.org/10.1016/j.beth.2009.08.002

28. Mosher CE, Secinti E, Li R, Hirsh AT, Bricker J, Miller KD, et al. Acceptance and commitment therapy for symptom interference in metastatic breast cancer patients: a pilot randomized trial. Support Care Cancer. 2018;26(6): 1993-2004. https://doi.org/10.1007/s00520-018-4045-0.

29. A-Tjak JGL, Davis ML, Morina N, Powers MB, Smits JAJ, Emmelkamp PMG. A meta-analysis of the efficacy of acceptance and commitment therapy for clinically relevant mental and physical health problems. Psychother Psychosom. 2015;84(1):30-6. https://doi.org/10.1159/000365764.

30. Hann KEJ, McCracken LM. A systematic review of randomized controlled trials of acceptance and commitment therapy for adults with chronic pain: outcome domains, design quality, and efficacy. J Contextual Behav Sci. 2014;3(4):217-27. https://doi.org/10.1016/j.jcbs.2014.10.001.

31. Hughes LS, Clark J, Colclough JA, Dale E, McMillan D. Acceptance and commitment therapy (ACT) for chronic pain: a systematic review and metaanalyses. Clin J Pain. 2017;33(6):552-68. https://doi.org/10.1097/AJP. 0000000000000425

32. Losada A, Marquez-Gonzalez M, Romero-Moreno R, Mausbach BT, Lopez J, Fernandez-Fernandez V, et al. Cognitive-behavioral therapy (CBT) versus acceptance and commitment therapy (ACT) for dementia family caregivers with significant depressive symptoms: results of a randomized clinical trial. J Consult Clin Psychol. 2015;83(4):760-72. https://doi.org/10.1037/ccp0000028.

33. Mosher CE, Secinti E, Hirsh AT, Hanna N, Einhorn LH, Jalal SI, et al. Acceptance and commitment therapy for symptom interference in advanced lung cancer and caregiver distress: a pilot randomized trial. J Pain Symptom Manag. 2019;58(4):632-44. https://doi.org/10.1016/j.jpainsymma n.2019.06.021.

34. Callahan CM, Unverzagt FW, Hui SL, Perkins AJ, Hendrie HC. Six-item screener to identify cognitive impairment among potential subjects for clinical research. Med Care. 2002;40(9):771-81. https://doi.org/10.1097/ 00005650-200209000-00007.

35. Bauer J, Capra S, Ferguson M. Use of the scored Patient-Generated Subjective Global Assessment (PG-SGA) as a nutrition assessment tool in patients with cancer. Eur J Clin Nutr. 2002;56(8):779-85. https://doi.org/10.1 038/sj.ejcn.1601412.

36. Hann DM, Denniston MM, Baker F. Measurement of fatigue in cancer patients: further validation of the Fatigue Symptom Inventory. Qual Life Res. 2000;9(7):847-54. https://doi.org/10.1023/A:1008900413113.

37. Hann DM, Jacobsen PB, Azzarello LM, Martin SC, Curran SL, Fields KK, et al. Measurement of fatigue in cancer patients: development and validation of the Fatigue Symptom Inventory. Qual Life Res. 1998;7(4):301-10. https://doi. org/10.1023/A:1008842517972.

38. Higginson IJ, Gao W, Jackson D, Murray J, Harding R. Short-form Zarit Caregiver Burden Interviews were valid in advanced conditions. J Clin Epidemiol. 2010;63(5):535-42. https://doi.org/10.1016/j.Ejclinepi.2009.06.014.

39. Pilkonis PA, Choi SW, Reise SP, Stover AM, Riley WT, Cella D. Item banks for measuring emotional distress from the Patient-Reported Outcomes Measurement Information System (PROMIS $\left.{ }^{(R)}\right)$ : depression, anxiety, and anger. Assessment. 2011;18(3):263-83. https://doi.org/10.1177/10731 91111411667. 
40. Van Cutsem E, Cervantes A, Nordlinger B, Arnold D. Metastatic colorectal cancer: ESMO Clinical Practice Guidelines for diagnosis, treatment and follow-up. Ann Oncol. 2014;25(suppl_3):iii1-9.

41. Snow G. blockrand: Randomization for block random clinical trials. $R$ package v 13. 2013.

42. Team RC. R: A language and environment for statistical computing. Vienna: R Foundation for Statistical Computing; 2014.

43. Butt Z, Parikh ND, Beaumont JL, Rosenbloom SK, Syrjala KL, Abernethy AP, et al. Development and validation of a symptom index for advanced hepatobiliary and pancreatic cancers: the National Comprehensive Cancer Network Functional Assessment of Cancer Therapy (NCCN-FACT) Hepatobiliary-Pancreatic Symptom Index (NFHSI). Cancer. 2012;118(23): 5997-6004. https://doi.org/10.1002/cncr.27588.

44. Mosher CE, Adams RN, Helft PR, O'Neil BH, Shahda S, Rattray NA, et al. Family caregiving challenges in advanced colorectal cancer: patient and caregiver perspectives. Support Care Cancer. 2016;24(5):2017-24. https://doi. org/10.1007/s00520-015-2995-z

45. Johns SA, Stutz PV, Talib TL, Cohee AA, Beck-Coon KA, Brown LF, et al. Acceptance and commitment therapy for breast cancer survivors with fear of cancer recurrence: a 3-arm pilot randomized controlled trial. Cancer. 2020:126(1):211-8. https://doi.org/10.1002/cncr.32518.

46. Mohabbat-Bahar S, Maleki-Rizi F, Akbari ME, Moradi-Joo M. Effectiveness of group training based on Acceptance and Commitment Therapy on anxiety and depression of women with breast cancer. Iran J Cancer Prev. 2015;8(2): 71-6.

47. Porter LS, Keefe FJ, Baucom DH, Hurwitz H, Moser B, Patterson E, et al. Partner-assisted emotional disclosure for patients with gastrointestinal cancer: results from a randomized controlled trial. Cancer. 2009;115(18 Suppl):4326-38. https://doi.org/10.1002/cncr.24578.

48. Mosher CE, Winger JG, Hanna N, Jalal SI, Einhorn LH, Birdas TJ, et al. Randomized pilot trial of a telephone symptom management intervention for symptomatic lung cancer patients and their family caregivers. J Pain Symptom Manag. 2016;52(4):469-82. https://doi.org/10.1016/j.jpainsymma n.2016.04.006.

49. Mosher CE, Secinti E, Johns SA, O'Neil BH, Helft PR, Shahda S, et al. Examining the effect of peer helping in a coping skills intervention: a randomized controlled trial for advanced gastrointestinal cancer patients and their family caregivers. Qual Life Res. 2018;27(2):515-28. https://doi. org/10.1007/s11136-017-1620-7

50. Bedard M, Molloy DW, Squire L, Dubois S, Lever JA, O'Donnell M. The Zarit Burden Interview: a new short version and screening version. Gerontologist. 2001;41(5):652-7. https://doi.org/10.1093/geront/41.5.652.

51. Buysse DJ, Yu L, Moul DE, Germain A, Stover A, Dodds NE, et al. Development and validation of patient-reported outcome measures for sleep disturbance and sleep-related impairments. Sleep. 2010;33(6):781-92. https://doi.org/10.1093/sleep/33.6.781

52. Yu L, Buysse DJ, Germain A, Moul DE, Stover A, Dodds NE, et al. Development of short forms from the PROMIS sleep disturbance and sleeprelated impairment item banks. Behav Sleep Med. 2012;10(1):6-24. https:// doi.org/10.1080/15402002.2012.636266.

53. Hahn EA, DeVellis RF, Bode RK, Garcia SF, Castel LD, Eisen SV, et al Measuring social health in the patient-reported outcomes measurement information system (PROMIS): item bank development and testing. Qual Life Res. 2010;19(7):1035-44. https://doi.org/10.1007/s11136-010-9654-0.

54. Smout M, Davies M, Burns N, Christie A. Development of the Valuing Questionnaire (VQ). J Contextual Behav Sci. 2014;3(3):164-72. https://doi. org/10.1016/j.jcbs.2014.06.001.

55. Bond FW, Hayes SC, Baer RA, Carpenter KM, Guenole N, Orcutt HK, et al. Preliminary psychometric properties of the Acceptance and Action Questionnaire-II: a revised measure of psychological inflexibility and experiential avoidance. Behav Ther. 2011;42(4):676-88. https://doi.org/10.101 6/j.beth.2011.03.007.

56. Cohen SR, Sawatzky R, Russell LB, Shahidi J, Heyland DK, Gadermann AM. Measuring the quality of life of people at the end of life: The McGill Quality of Life Questionnaire-Revised. Palliat Med. 2017;31(2):120-9. https://doi. org/10.1177/0269216316659603.

57. Hays RD, Bjorner JB, Revicki DA, Spritzer KL, Cella D. Development of physical and mental health summary scores from the patient-reported outcomes measurement information system (PROMIS) global items. Qual Life Res. 2009;18(7):873-80. https://doi.org/10.1007/s11136-009-9496-9.
58. Kroenke K, Bair M, Damush T, Hoke S, Nicholas G, Kempf C, et al. Stepped Care for Affective Disorders and Musculoskeletal Pain (SCAMP) study: design and practical implications of an intervention for comorbid pain and depression. Gen Hosp Psychiatry. 2007;29(6):506-17. https://doi.org/10.1016/ j.genhosppsych.2007.08.005.

59. Kroenke K, Theobald D, Norton K, Sanders R, Schlundt S, McCalley S, et al. The Indiana Cancer Pain and Depression (INCPAD) trial: design of a telecare management intervention for cancer-related symptoms and baseline characteristics of study participants. Gen Hosp Psychiatry. 2009;31(3):240-53. https://doi.org/10.1016/j.genhosppsych.2009.01.007.

60. Kroenke K, Theobald D, Wu J, Norton K, Morrison G, Carpenter J, et al. Effect of telecare management on pain and depression in patients with cancer: a randomized trial. JAMA. 2010;304(2):163-71. https://doi.org/10.1001/jama.2 010.944.

61. Kadan-Lottick NS, Vanderwerker LC, Block SD, Zhang B, Prigerson HG Psychiatric disorders and mental health service use in patients with advanced cancer. Cancer. 2005;104(12):2872-81. https://doi.org/10.1002/ cncr.21532.

62. Vanderwerker LC, Laff RE, Kadan-Lottick NS, McColl S, Prigerson HG. Psychiatric disorders and mental health service use among caregivers of advanced cancer patients. J Clin Oncol. 2005;23(28):6899-907. https://doi. org/10.1200/JCO.2005.01.370.

63. Kroenke K, Krebs E, Wu J, Bair MJ, Damush T, Chumbler N, et al. Stepped Care to Optimize Pain care Effectiveness (SCOPE) trial study design and sample characteristics. Contemp Clin Trials. 2013;34(2):270-81. https://doi. org/10.1016/j.cct.2012.11.008

64. Kroenke K, Evans E, Weitlauf S, McCalley S, Porter B, Williams T, et al. Comprehensive vs. Assisted Management of Mood and Pain Symptoms (CAMMPS) trial: study design and sample characteristics. Contemp Clin Trials. 2018;64:179-87. https://doi.org/10.1016/j.cct.2017.10.006.

65. Perkins AJ, Kroenke K, Unützer J, Katon W, Williams JW, Hope C, et al. Common comorbidity scales were similar in their ability to predict health care costs and mortality. J Clin Epidemiol. 2004;57(10):1040-8. https://doi. org/10.1016/j.jclinepi.2004.03.002.

66. Cella D, Riley W, Stone A, Rothrock N, Reeve B, Yount S, et al. The PatientReported Outcomes Measurement Information System (PROMIS) developed and tested its first wave of adult self-reported health outcome item banks: 2005-2008. J Clin Epidemiol. 2010;63(11):1179-94. https://doi.org/10.1016/j. jclinepi.2010.04.011.

67. Jensen RE, Potosky AL, Moinpour CM, Lobo T, Cella D, Hahn EA, et al. United States population-based estimates of Patient-Reported Outcomes Measurement Information System symptom and functional status reference values for individuals with cancer. J Clin Oncol. 2017;35(17):1913-20. https:// doi.org/10.1200/JCO.2016.71.4410.

68. Choi SW, Reise SP, Pilkonis PA, Hays RD, Cella D. Efficiency of static and computer adaptive short forms compared to full-length measures of depressive symptoms. Qual Life Res. 2010;19(1):125-36. https://doi.org/10.1 007/s11136-009-9560-5.

69. Amtmann D, Cook KF, Jensen MP, Chen W-H, Choi S, Revicki D, et al. Development of a PROMIS item bank to measure pain interference. Pain. 2010;150(1):173-82. https://doi.org/10.1016/j.pain.2010.04.025.

70. Jakobsen JC, Gluud C, Wetterslev J, Winkel P. When and how should multiple imputation be used for handling missing data in randomised clinical trials - a practical guide with flowcharts. BMC Med Res Methodol. 2017;17(1):162. https://doi.org/10.1186/s12874-017-0442-1.

71. Altman DG. Comparability of randomised groups. J R Stat Soc Series D Stat. 1985;34(1):125-36.

72. Badr H, Smith CB, Goldstein NE, Gomez JE, Redd WH. Dyadic psychosocial intervention for advanced lung cancer patients and their family caregivers: results of a randomized pilot trial. Cancer. 2015;121(1):150-8. https://doi. org/10.1002/cncr.29009.

73. Borkan J. Immersion/crystallization. In: Crabtree BF, Miller WL, editors. Doing qualitative research. 2nd ed. Thousand Oaks: Sage; 1999. p. 179-94.

74. Lindlof TR, Taylor BC. Qualitative communication research methods. 2nd ed. Thousand Oaks: Sage; 2002.

75. Sim J. Should treatment effects be estimated in pilot and feasibility studies? Pilot Feasib Stud. 2019;5(1):107. https://doi.org/10.1186/s40814-019-0493-7.

76. Atkins DC. Using multilevel models to analyze couple and family treatment data: basic and advanced issues. J Fam Psychol. 2005;19(1):98-110. https:// doi.org/10.1037/0893-3200.19.1.98. 
77. Kenny D, Kashy D, Cook W. Dyadic data analysis. New York: Guilford Press; 2006.

78. Rounsaville BJ, Carroll KM, Onken LS. A stage model of behavioral therapies research: getting started and moving on from stage I. Clin Psychol: Sci Pract. 2001;8(2):133-42. https://doi.org/10.1093/clipsy.8.2.133.

79. Dong N, Maynard R. PowerUp!: A tool for calculating minimum detectable effect sizes and minimum required sample sizes for experimental and quasiexperimental design studies. J Res Educ Eff. 2013;6(1):24-67.

80. Butt Z, Rosenbloom SK, Abernethy AP, Beaumont JL, Paul D, Hampton D, et al. Fatigue is the most important symptom for advanced cancer patients who have had chemotherapy. J Natl Compr Canc Netw. 2008;6(5):448-55. https://doi.org/10.6004/jnccn.2008.0036.

81. Bower JE. Treating cancer-related fatigue: The search for interventions that target those most in need. J Clin Oncol. 2012;30(36):4449-50. https://doi. org/10.1200/JCO.2012.46.0436.

\section{Publisher's Note}

Springer Nature remains neutral with regard to jurisdictional claims in published maps and institutional affiliations.

Ready to submit your research? Choose BMC and benefit from:

- fast, convenient online submission

- thorough peer review by experienced researchers in your field

- rapid publication on acceptance

- support for research data, including large and complex data types

- gold Open Access which fosters wider collaboration and increased citations

- maximum visibility for your research: over $100 \mathrm{M}$ website views per year

At BMC, research is always in progress.

Learn more biomedcentral.com/submissions 